\title{
On a Paradoxical Situation Related to Bonded Joints: Could Stiffer Mid-Portions of a Compliant Attachment Result in Lower Thermal Stress?*
}

\author{
Ephraim SUHIR $^{* *}$ \\ ** University of California, Santa Cruz, CA, University of Maryland, College Park, MA, and \\ ERS Co., Los Altos, CA, USA, 727 Alvina Ct., Los Altos, CA 94024, E-mail:suhire@aol.com
}

\begin{abstract}
The objective of the analysis is to find out to what extent the thermal stress in a bonded assembly could be reduced as a result of employing a compliant bonding layer with an interfacial compliance varying along the assembly. It has been determined that by employing such a layer one can achieve in an assembly of any size a low stress level and the same type of the stress distribution that takes place in a small size assembly: the interfacial shearing stress changes linearly along the assembly and the normal stresses in the adherends follow a parabolic law. We have encountered a rather paradoxical situation taking place in an assembly with an optimized longitudinal distribution of the interfacial compliance (that increases in accordance with a parabolic law from a next-to-zero value in the mid-portion of the assembly to large values at its ends): the maximum stress in such an assembly is lower than the maximum stress in an assembly with a highly compliant bonding layer of constant compliance of the same magnitude as the (high) compliance at the ends of an assembly with an optimized compliance. We conclude that this situation occurs because the peripheral interfacial displacements in an assembly with the varying compliance are reduced owing to the stiffer (less compliant) mid-portion of the bonding layer.
\end{abstract}

Key words: Bonded Joints, Thermal Stress, Interfacial Stress, Compliant Attachments (Bonds)

\section{Introduction}

Bonded assemblies subjected to thermal loading are widely used in electronics and photonics $^{1-13}$. It has been established ${ }^{8}$ that the thermal stress in small-size assemblies can be significantly lower than in large assemblies, and could be further reduced by employing a compliant bonding layer. In this case, the interfacial shearing stress becomes distributed linearly over the assembly length, while the forces (normal stresses) in the adherends change in accordance with a parabolic law. The governing parameter, as far as the magnitude and the distribution of the induced elastic stresses are concerned, is the product $k a$ of the parameter $k=\sqrt{\frac{\lambda}{\kappa}}$ of the interfacial shearing stress and half the assembly size $a$. Here $\lambda$ is the total axial compliance of the assembly, and $\kappa$ is its total interfacial compliance. The effect of the assembly size and the attachment compliance becomes significant for $k a \leq 2.5$. If this value is exceeded, the further increase in the $k a$ value does not lead to an increase in the stress level. In conventional assemblies characterized by a thin and/or low modulus bonding layer the total axial compliance $\lambda=\lambda_{1}+\lambda_{2}$ is due 
to the compliances $\lambda_{1}$ and $\lambda_{2}$ of the adherends only, while the total interfacial compliance $\kappa=\kappa_{0}+\kappa_{1}+\kappa_{2}$ is due to both the compliances of the adherends $\left(\kappa_{1}+\kappa_{2}\right)$ and the bonding layer $\left(\kappa_{0}\right)$. In assemblies with a highly compliant bonding layer, it is its compliance $\kappa_{0}$ that plays the prevailing role.

In the analysis that follows we intend to find out to what extent the thermal stress in a bonded assembly could be reduced as a result of employing a bonding layer of high interfacial compliance that, in addition, varies along the assembly. Particularly, we are going to determine if by employing a bonding layer of high and variable compliance one could achieve in an assembly of any size the same type of the stress distribution that takes place in a small size assembly: the interfacial shearing stress changes linearly along the assembly, and the normal stresses in the adherends follow a parabolic law. If this occurs it is expected that the maximum stress will be brought down appreciably.

\section{Analysis}

\section{Basic Equation}

Let a bi-material assembly be manufactured at an elevated temperature and subsequently cooled down to a low temperature. The interfacial longitudinal displacements, $u_{1}(x)$ and $u_{2}(x)$, of the assembly components \#1 and \#2 (Fig.1) could be sought, in an approximate analysis, as follows ${ }^{8}$ :

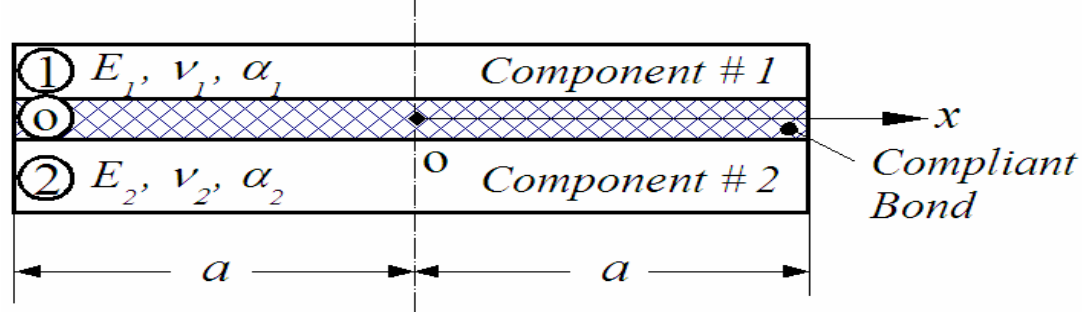

Fig. 1. Small size assembly with a compliant bonding layer ( $E=$ Young's modulus, $v=$ Poison's ratio, $a=$ coefficient of thermal expansion).

$$
\left.\begin{array}{l}
u_{1}(x)=-\alpha_{1} \Delta t x+\lambda_{1} \int_{0}^{x} T(\xi) d \xi-\kappa_{1} \tau(x) \\
u_{2}(x)=-\alpha_{2} \Delta t x-\lambda_{2} \int_{0}^{x} T(\xi) d \xi+\kappa_{2} \tau(x)
\end{array}\right\} .
$$

Here $\alpha_{1}$ and $\alpha_{2}$ are the coefficients of thermal expansion (CTE) of the adherend materials, $\Delta t$ is the change in temperature,

$$
\lambda_{1}=\frac{1-v_{1}}{E_{1} h_{1}}, \quad \lambda_{2}=\frac{1-v_{2}}{E_{2} h_{2}}
$$

are the axial compliances of the assembly components, $h_{1}$ and $h_{2}$ are their thicknesses, $E_{1}$ and $E_{2}$ are Young's moduli of the materials, $v_{1}$ and $v_{2}$ are Poisson's ratios,

$$
T(x)=\int_{-a}^{x} \tau(\xi) d \xi
$$

is the force acting in the $x$ cross-section, $\tau(x)$ is the interfacial shearing stress, $a$ is half the assembly length, and $\kappa_{1}$ and $\kappa_{2}$ are the interfacial compliances of the assembly components $^{8,10}$. The origin of the coordinate $x$ is in the mid-cross-section of the assembly.

The first terms in (1) are stress-free thermal contractions. The second terms determine 
the displacements due to the induced thermal forces and are evaluated in accordance with the Hooke's law. The third terms account for the fact that the interfacial displacements are somewhat larger than the displacements of the inner points of the cross-section.

The condition of the compatibility of the displacements (1) can be written as

$$
u_{1}(x)=u_{2}(x)+\kappa_{0} \tau(x) .
$$

where $\kappa_{0}$ is the longitudinal compliance of the bonding layer. Introducing the formulas (1) into this condition we obtain the following basic equation for the shearing stress function $\tau(x)$ :

$$
\kappa \tau(x)-\lambda \int_{0}^{x} T(\xi) d \xi=\Delta \alpha \Delta t x .
$$

Here $\kappa=\kappa_{0}+\kappa_{1}+\kappa_{2}$ is the total interfacial compliance of the assembly, $\lambda=\lambda_{1}+\lambda_{2}$ is its total axial compliance, and $\Delta \alpha=\alpha_{2}-\alpha_{1}$ is the thermal expansion (contraction) mismatch of the components' materials.

\section{Constant Interfacial Compliance}

Let us assume first that the compliance $\kappa_{0}$ of the bonding layer is the same throughout the assembly. Since the shearing stress $\tau(x)$ is supposed to be axisymmetric with respect to the origin, the solution to the equation $(5)$ is

$$
\tau(x)=C \sinh k x \text {. }
$$

Then the formula (3) yields:

$$
T(x)=-\frac{C}{k}(\cosh k a-\cosh k x) .
$$

Introducing (6) and (7) into the equation (5), we conclude that this equation is fulfilled if the following relationship takes place:

$$
\left(\kappa-\frac{\lambda}{k^{2}}\right) C \sinh k x+\left(\frac{\lambda}{k} C \cosh k a-\Delta \alpha \Delta t\right) x=0 .
$$

This relationship, in its turn, will be fulfilled for any $x$ value, if

$$
k=\sqrt{\frac{\lambda}{\kappa}}, \quad C=k \frac{\Delta \alpha \Delta t}{\lambda} \frac{1}{\cosh k a} .
$$

Then the formulas (6) and (7) yield:

$$
\tau(x)=k \frac{\Delta \alpha \Delta t}{\lambda} \frac{\sinh k x}{\cosh k a}, \quad T(x)=-\frac{\Delta \alpha \Delta t}{\lambda}\left(1-\frac{\cosh k x}{\cosh k a}\right) .
$$

The relative longitudinal displacement of the assembly components can be found as

$$
\Delta u(x)=\kappa \tau(x)=\frac{\Delta \alpha \Delta t}{k} \frac{\sinh k x}{\cosh k a} .
$$

The maximum shearing stress and the maximum relative displacement occur at the assembly ends $x= \pm a$ :

$$
\tau_{\max }=k \frac{\Delta \alpha \Delta t}{\lambda} \tanh k a, \quad \Delta u_{\max }=\kappa \tau_{\max }=\frac{\Delta \alpha \Delta t}{k} \tanh k a .
$$

The maximum thermally induced force occurs in the mid-cross-section:

$$
T_{\max }=-\frac{\Delta \alpha \Delta t}{\lambda}\left(1-\frac{1}{\cosh k a}\right) .
$$

In the case of long assemblies (large $a$ ) and/or assemblies with compliant adherends and a stiff bonding layer (large $\lambda$ and/or low $\kappa$ and, hence, large $k$ ), when the parameter $k a \geq 2.5$, the formulas (10)-(13) can be simplified as follows: 


$$
\begin{gathered}
\tau(x)=\tau_{\max } e^{-k(a-x)}, \quad \tau_{\max }=\tau_{\max }^{\infty}=k \frac{\Delta \alpha \Delta t}{\lambda}=k T_{\max }^{\infty}, \\
T(x)=-T_{\max }\left(1-e^{-k(a-x)}\right), T_{\max }=T_{\max }^{\infty}=\frac{\Delta \alpha \Delta t}{\lambda}, \\
\Delta u(x)=u_{\text {max }} e^{-k(a-x)}, \quad u_{\text {max }}=u_{\text {max }}^{\infty}=\frac{\Delta \alpha \Delta t}{k}=\kappa \tau_{\text {max }}^{\infty} .
\end{gathered}
$$

In the case of small-size assemblies (small $a$ ) and/or assemblies with stiff adherends and a compliant bonding layer (small $\lambda$ and/or large $\kappa$ and, hence, small $k$ ), when $k a \leq 0.25$, the formulas (10)-(13) yield:

$$
\begin{array}{r}
\tau(x)=\tau_{\max } \frac{x}{a}, \quad \tau_{\max }=\frac{\Delta \alpha \Delta t}{\kappa} a=k a \tau_{\max }^{\infty}, \\
T(x)=-T_{\max }\left(1-\frac{x^{2}}{a^{2}}\right), \quad T_{\max }=\frac{\Delta \alpha \Delta t}{2 \kappa} a^{2}=\frac{1}{2}(k a)^{2} T_{\max }^{\infty}, \\
\Delta u(x)=\kappa \tau(x)=\Delta u_{\max } \frac{x}{a}, \quad \Delta u_{\max }=a \Delta \alpha \Delta t=k a u_{\text {max }}^{\infty} .
\end{array}
$$

Thus, the stresses and the displacements could be very small, if the product $k a$ is below 0.25 and is small, i.e., much smaller than 0.25 . Could a similar effect be achieved if a bonding layer of variable longitudinal interfacial compliance $\kappa_{0}$ is employed?

\section{Variable Interfacial Compliance}

Let us find out now if there exist a distribution $\kappa_{0}=\kappa_{0}(x)$ of the longitudinal interfacial compliance $\kappa_{0}$ of the bonding layer that results in a linearly distributed interfacial shearing stress

$$
\tau(x)=C x
$$

If there is, then the forces in the adherend cross-sections would be

$$
T(x)=-\frac{1}{2} C\left(a^{2}-x^{2}\right) .
$$

In such a case the equation (5) yields (for $x \neq 0$ ) :

$$
\left[\kappa(x)+\frac{1}{2} \lambda a^{2}-\frac{1}{6} \lambda x^{2}\right] C=\Delta \alpha \Delta t .
$$

This equation is fulfilled if the interfacial compliance is expressed as

$$
\kappa(x)=\kappa_{1}+\kappa_{2}+\kappa_{0}(a) \frac{x^{2}}{a^{2}} .
$$

Then the equation (22) yields:

$$
C=\frac{\Delta \alpha \Delta t}{\kappa_{1}+\kappa_{2}+3 \kappa_{0}(a)}, \kappa_{0}(a)=\frac{1}{6} \lambda a^{2},
$$

and therefore the following relationships should take place:

$$
\begin{aligned}
& \kappa(x)=\kappa_{0}(x)+\kappa_{1}+\kappa_{2}, \quad \kappa_{0}(x)=\frac{1}{6} \lambda x^{2}, \\
& \tau(x)=\tau_{\max } \frac{x}{a}, \quad \tau_{\max }=\frac{\Delta \alpha \Delta t}{\kappa_{1}+\kappa_{2}+3 \kappa_{0}(a)} a, \\
& T(x)=-T_{\max }\left(1-\frac{x^{2}}{a^{2}}\right), \quad T_{\max }=\frac{1}{2} \frac{\Delta \alpha \Delta t}{\kappa_{1}+\kappa_{2}+3 \kappa_{0}(a)} a^{2},
\end{aligned}
$$




$$
\Delta u(x)=\Delta u_{\max } \frac{\kappa_{0}(x)+\kappa_{1}+\kappa_{2}}{\kappa_{0}(l)+\kappa_{1}+\kappa_{2}} \frac{x}{a}, \Delta u_{\max }=\frac{\kappa_{0}(a)+\kappa_{1}+\kappa_{2}}{3 \kappa_{0}(a)+\kappa_{1}+\kappa_{2}} a \Delta \alpha \Delta t .
$$

Comparing the second formulas in (26) and in (17) we conclude that the ratio of the maximum interfacial shearing stress in an assembly with a variable longitudinal interfacial compliance of the bonding layer to the maximum interfacial shearing stress in a small size assembly with a constant (but still large) longitudinal interfacial compliance $\kappa_{0}^{*}$ of this layer is expressed as

$$
\chi_{1}=\frac{\kappa_{0}^{*}+\kappa_{1}+\kappa_{2}}{3 \kappa_{0}(a)+\kappa_{1}+\kappa_{2}} .
$$

Thus formula indicates that even if the entire bonding layer in an assembly with the constant interfacial compliance has the same interfacial compliance as the maximum compliance of the bonding layer of variable compliance $\left(\kappa_{0}^{*}=\kappa_{0}(a)\right)$, the maximum interfacial shearing stress will be still lower in an assembly with a variable compliance of the bonding layer. The ratio $\chi_{1}$ will be the lowest, if the assembly components are characterized by low interfacial compliances compared to the compliance of the bonding layer:

$$
\chi_{1}=\frac{\kappa_{0}^{*}}{3 \kappa_{0}(a)}
$$

Thus, if this is the case, the compliance of the bonding layer of constant compliance should be three times as high as the maximum compliance of the bonding layer with variable compliance in order to result in the same maximum shearing stress. This paradoxical situation is due to the fact that the peripheral longitudinal displacements are considerably larger in assemblies with bonding layers of constant compliance. Indeed, comparing the second formula in (28) with the second formula in (19) we conclude that the maximum longitudinal relative interfacial displacement in an assembly with a bonding layer of constant compliance should be multiplied by the reduction factor of $\chi_{1}$ to obtain the maximum relative displacement of the assembly components in the case of a bonding layer of variable compliance. So, it is desirable, from the standpoint of the possible stress relief in the shearing stress at the assembly ends, to increase the interfacial compliance of the assembly only at its peripheral portions, while keeping the interfacial compliance in its mid-portion at a low enough level. Note that a similar rationale has been, in effect, utilized earlier in assemblies with a high modulus bonding material in the mid-portion of the assembly and low modulus adhesive at the assembly ends ${ }^{11-14}$. Obviously, if the interfacial compliances $\kappa_{1}$ and $\kappa_{2}$ of the assembly components are significantly larger than the compliance $\kappa_{0}$ of the bonding layer, there is no incentive for using bonding layers of elevated or variable compliance. There is no such an incentive also in a situation, when the assembly is characterized by a $k a$ value well below 0.25 , i.e., when the induced stresses are low anyway.

We would like to point out that the results of the above analysis will be different, at least from the quantitative point of view, if the bonding material exhibits plastic and/or time dependent deformations. Although the case of an inelastic and/or time-dependent behavior of the bonding material is beyond the scope of this analysis, we would like to point out that an approach of the type taken in the Ref.12 could be used to address such a case. In any event, an elastic analysis considered in this article should precede any inelastic analysis, if the latter is pursued.

\section{Numerical Example}

\section{Input Data:}

Half assembly length: $a=2.5 \mathrm{~mm}$, Change in temperature: $\Delta t=220^{\circ} \mathrm{C}$. 
Component \#1: Young's modulus: $E_{1}=169 \mathrm{GPa}=17250 \mathrm{~kg} / \mathrm{mm}^{2}$, Poisson's ratio: $v_{1}=0.24$, CTE: $\alpha_{1}=2.6 \times 10^{-6} 1 /{ }^{0} C$, Thickness: $h_{1}=0.25 \mathrm{~mm}$; Component \#2: Young's modulus: $E_{2}=85 G P a=8676 \mathrm{~kg} / \mathrm{mm}^{2}$, Poisson's ratio: $v_{2}=0.32$, CTE: $\alpha_{2}=16 \times 10^{-6} 1 /^{0} \mathrm{C}, h_{2}=0.5 \mathrm{~mm}$.

\section{Computed Data:}

Applied thermal strain: $\Delta \alpha \Delta t=0.002948$. Axial Compliances: $\lambda_{1}=\frac{1-v_{1}}{E_{1} h_{1}}=1.7623 \times 10^{-4} \mathrm{~mm} / \mathrm{kg} \quad, \quad \lambda_{2}=\frac{1-v_{2}}{E_{2} h_{2}}=1.5675 \times 10^{-4} \mathrm{~mm} / \mathrm{kg}$, $\lambda=\lambda_{1}+\lambda_{2}=3.3298 \times 10^{-4} \mathrm{~mm} / \mathrm{kg}$, Shear moduli : $G_{1}=6956 \mathrm{~kg} / \mathrm{mm}^{2}$, $G_{2}=3286 \mathrm{~kg} / \mathrm{mm}^{2}$

\section{Bonding Laver of Constant Compliance}

Bonding layer: Young's modulus: $E_{0}=2.5 \mathrm{GPa}=255.2 \mathrm{~kg} / \mathrm{mm}^{2}$, Poisson's ratio: $v_{0}=0.40$, Thickness $h_{0}=0.050 \mathrm{~mm}$, Shear modulus: $G_{0}=91.143 \mathrm{~kg} / \mathrm{mm}^{2}$ Interfacial compliances :

$$
\begin{aligned}
& \kappa_{1}=\frac{h_{1}}{3 G_{1}}=11.9801 \times 10^{-6} \mathrm{~mm}^{3} / \mathrm{kg}, \kappa_{2}=\frac{h_{2}}{3 G_{2}}=50.7202 \times 10^{-6} \mathrm{~mm}^{3} / \mathrm{kg}, \\
& \kappa_{0}=\frac{h_{0}}{G_{0}}=548.588 \times 10^{-6} \mathrm{~mm}^{3} / \mathrm{kg}, \\
& \kappa=\kappa_{0}+\kappa_{1}+\kappa_{2}=611.2888 \times 10^{-6} \mathrm{~mm}^{3} / \mathrm{kg}
\end{aligned}
$$

Parameters of the interfacial shearing stress and the governing parameter of the assembly length:

$k=0.7380 \mathrm{~mm}^{-1}, \quad k a=1.8450$.

Maximum thermally induced force:

$T_{\max }=-\frac{\Delta \alpha \Delta t}{\lambda}\left(1-\frac{1}{\cosh k a}\right)=6.1234 \mathrm{~kg} / \mathrm{mm}$

Maximum interfacial shearing stress: $\tau_{\max }=k \frac{\Delta \alpha \Delta t}{\lambda} \tanh \mathrm{ka}=8.4218 \mathrm{~kg} / \mathrm{mm}^{2}$

Maximum relative displacement: $\Delta u_{\max }=\kappa \tau_{\max }=5.1481 \mu m$

\section{Bonding Layer of Variable Compliance.}

Maximum interfacial compliance at the assembly end:

$\kappa_{0}=548.588 \times 10^{-6} \mathrm{~mm}^{3} / \mathrm{kg}$

Maximum thermally induced force:

$T_{\max }=\frac{1}{2} \frac{\Delta \alpha \Delta t}{\kappa_{1}+\kappa_{2}+3 \kappa_{0}(l)} a^{2}=5.3923 \mathrm{~kg} / \mathrm{mm}$

Maximum shearing stress: $\tau_{\max }=\frac{\Delta \alpha \Delta t}{\kappa_{1}+\kappa_{2}+3 \kappa_{0}(a)} a=4.3138 \mathrm{~kg} / \mathrm{mm}^{2}$

Maximum relative displacement: $\Delta u_{\max }=\frac{\kappa_{0}(a)+\kappa_{1}+\kappa_{2}}{3 \kappa_{0}(a)+\kappa_{1}+\kappa_{2}} a \Delta \alpha \Delta t=2.6370 \mu \mathrm{m}$

If the maximum compliance at the assembly end is doubled $\left(\kappa_{0}=2 \times 548.588 \times 10^{-6}=1097.176 \times x 10^{-6} \mathrm{~mm}^{3} / \mathrm{kg}\right)$ then the above maximum thermally induced force and the maximum interfacial shearing stress would occur in assemblies with $a=3.503 \mathrm{~mm}$ and $a=4.908 \mathrm{~mm}$, respectively. 


\section{Conclusions}

A simple and physically meaningful model has been developed for the evaluation of the thermally induced stresses in a bonded assembly with an attachment layer of variable longitudinal interfacial compliance. We have determined that if the longitudinal interfacial compliance of the bonding layer increases with an increase in the distance from the mid-cross-section of the assembly squared, then the stresses in an assembly of any size will be distributed similarly to the distribution of such stresses in small-size assemblies with a bonding layer of constant compliance, i.e., the shearing stress will be distributed linearly over the assembly length and the normal stresses in the cross-sections of the assembly components will be described by a quadratic parabola. However, if the maximum interfacial compliance at the end of an assembly with a bonding layer of variable compliance is not very large and, in particular, not larger than the compliance of the layer of constant longitudinal compliance, then an appreciable stress relief can be achieved only in small size assemblies, while in large size assemblies these stresses can be even higher than in assemblies with stiff interfaces.

We have encountered a rather paradoxical situation in assemblies with attachments of appreciable and variable compliance : the maximum stresses in an assembly with an interfacial compliance of the bonding layer that increases in accordance with a quadratic law from a next-to-zero value at the mid-cross-section of the assembly to its maximum value at the assembly end are lower than the stresses in an assembly with a bonding layer of constant compliance of the same magnitude as the maximum compliance at the end of an assembly with an attachment layer of variable compliance. This paradoxical situation is explained by the fact that in the latter assembly the longitudinal interfacial displacements are reduced owing to the stiffer mid-portions of the compliant attachment.

In the numerical example carried out for a $5 \mathrm{~mm}$ large assembly, an almost $50 \%$ relief in the maximum interfacial shearing stress and a $50 \%$ reduction in the maximum relative displacements (at the assembly ends) has been achieved for an assembly with the bonding layer of variable compliance and with a stiff mid-portion compared to the case of an assembly with the constant interfacial compliance of the same magnitude as the compliance at the end of the assembly with the variable compliance. The predicted relief in the maximum force (normal stress) in the mid-cross-sections of the adherends is about $12 \%$. In practice, variable longitudinal interfacial compliance can be obtained by using materials with lower Young's moduli at the peripheral portions of the assembly and/or by changing the thickness of the bonding layer (say, as a result of slanting the assembly components at the assembly ends).

\section{References}

1. R. Zeyfang, "Stresses and Strains in a Plate Bonded to a Substrate: Semiconductor Devices", Solid State Electronics, vol.14, 1971

2. P.B.Grimado, "Interlaminated Thermo-Elastic Stresses in Layered Beams", J. Therm. Stresses, vol. 1, 1978.

3. W.T. Chen and C.W.Nelson, "Thermal Stresses in Bonded Joints", IBM Journal, Research and Development, vol.23, No.2, 1979.

4. D. Chen, S. Cheng, T.D.Geerhardt, "Thermal Stresses in Laminated Beams", J. Thermal Stresses, vol.5, 1982.

5. F.-V. Chang, "Thermal Contact Stresses of Bi-Metal Strip Thermostat", Applied Mathematics and Mechanics, vol.4, No.3, Tsing-hua Univ., Beijing, China, 1983.

6. S. E. Yamada, "A Bonded Joint Analysis for Surface Mount Components", ASME Journal of Electronic Packaging, vol.114, No.1, 1992

7. J.H.Lau, ed., "Thermal Stress and Strain in Microelectronics Packaging”, Van-Nostrand Reinhold, New York, 1993. 
8. E. Suhir, "Stresses in Bi-Metal Thermostats", ASME Journal of Applied Mechanics, vol. 53, No. 3, Sept. 1986.

9. E. Suhir, "Thermal Stress in a Bi-Material Assembly Adhesively Bonded at the Ends", Journal of Applied Physics, vol. 89, No.1, 2001.

10. E. Suhir, "Bi-Material Assembly Adhesively Bonded at the Ends and Fabrication Method", US Patent \#6,460, 753, 2002

11. E. Suhir, "Thermal Stress in a Polymer Coated Optical Glass Fiber with a Low Modulus Coating at the Ends", Journal of Materials Research, vol. 16, No. 10, 2001

12. E. Suhir, "Thermal Stress in an Adhesively Bonded Joint with a Low Modulus Adhesive Layer at the Ends", Journal of Applied Physics, April 2003.

13. E. Suhir, "Thermal Stress in a Bi-Material Assembly with a "Piecewise-Continuous" Bonding Layer: Theorem of Three Axial Forces", Journal of Applied Physics, D, 42, 2009 